\title{
Estudio geológico de suelos en el sector Vinchoa de la parroquia Veintimilla del cantón Guaranda
}

\author{
Jorge Luis Poma Taris \\ Universidad Central del Ecuador \\ jorgepoma2006@yahoo.com
}

Recibido: 01 de septiembre de 2017 / Aprobado: 30 de noviembre de 2017

\section{Resumen}

El proyecto realizado en el sector de la parroquia Veintimilla del cantón Guaranda consistió en el estudio geológico de suelos, basado en la caracterización de suelos desde el punto de vista geológico, analizar sus características físicas, geológicas y morfológicas. Para la identificación de los tipos de suelos se hizo calicatas, con una profundidad de $1.5 \mathrm{~m}$, en las que se identificaron tres tipos de horizontes de suelos, de donde se obtuvieron muestras, encontrándose un $\mathrm{pH}$ de 6,18 considerados suelos ácidos.

Como referencia, los suelos de Vinchoa, una de las comunidades investigadas poseen los siguientes porcentajes de contenido: arena $62,8 \%$, limo $36,0 \%$ y arcilla $1,2 \%$; densidad $2,24 \mathrm{~g} / \mathrm{cm}^{3}$.

El terreno analizado cumpliría con la exigencia de especificidad de terreno apto para el uso agrícola y edificaciones.

La morfología de la zona se caracteriza por ser de relieves no muy inclinados predominando pendientes en el orden del $5 \%$ al $70 \%$.

Palabras clave: suelo sedimentario, horizontes del suelo, calicatas, apertura de calicatas, muestreo de suelo. 


\section{Abstract}

The project realized at Veintimilla's Parish in Guaranda canton consisted in the geological study of soils, based on the characterization of soils from the geological point of view, it also analyzes their physical, geological and morphological characteristics.

Gauges were made in order to identify soil types, with a depth of $1.5 \mathrm{~m}$, identifying three types of soil horizons. Samples were obtained with a $\mathrm{pH}$ of 6.18 considered as acid soils due to the scarcity of forests.

As a reference in Vinchoa, the soil of one of the communities has the following percentages: sand content $62,8 \%$, sludge $36,0 \%$ and clay $1,2 \%$, density $2.24 \mathrm{~g} / \mathrm{cm}^{3}$. The analyzed soils would meet the specific requirements of suitable soil for agricultural use and buildings.

The morphology of the area is characterized by not very steep slopes, predominating slopes in the order of $5 \%$ to $70 \%$.

Keywords: sedimentary soil, soil horizons, calicates, calicate opening, soil sampling. 


\section{Introducción}

S e han realizado varios estudios y trabajos prácticos mediante el análisis de suelos utilizando tecnologías y técnicas cada vez más avanzadas, priorizando la caracterización del medio físico para la planificación por la necesidad de relevar los recursos del suelo, con la finalidad de identificar las áreas ambientalmente frágiles o sensibles a sufrir afectaciones por fenómenos naturales o antrópicos (Brizuela, 2013).

El estudio de suelos del sector Vinchoa, se realizó en base a interpretación de información cartográfica y estadística existente en las diferentes instituciones locales y nacionales tales como MAGAP, Ministerio del Ambiente, Municipio de Guaranda, investigación de campo, entre otros, los mismos que permitieron establecer los grados de intervención del hombre en el lugar de interés, además de identificar las principales amenazas, la combinación de las acciones naturales o antrópicas sobre el medio físico en donde se emplaza la parroquia Veintimilla. (Guaranda, 2014).

Los estudios y análisis de suelos nos permiten aportar conocimientos detallados de zonas que presentan características físicas, geológicas y morfológicas, las mismas que deben interpretarse, evaluarse, analizarse desde el punto de vista geológico.

La comunidad Vinchoa perteneciente a la parroquia Veintimilla, está ubicada en las estribaciones de la cordillera Occidental considerada tectónicamente activa, lo cual hace que se exponga a amenazas de origen natural de diferente índole, erupciones volcánicas, sismos, movimientos dinámicos del terreno como erosión, reptación.

La característica geomorfológica de la zona está marcada por la presencia de cerros de mediana altura, de relieve irregular, y pendientes medias; la mayor parte presenta una mecánica de inestabilidad, gran parte de los suelos son poco consolidados en el sentido geológico. (Guaranda, 2014).

La ampliación de la frontera agrícola y la tala indiscriminada de árboles es un factor que ha favorecido los fenómenos de movimientos en masa.

La evaluación del estudio de suelos se realiza en base a sus características físicas, geológicas y morfológicas, lo que nos permite desarrollar un estudio con mayor enfoque en sus características.

En consecuencia, el objetivo de la investigación se cumplirá de manera exitosa, contribuyendo al mejoramiento de la práctica desarrollada en el campo.

\section{Alcance}

Para la investigación y estudio geológico de suelos se va a analizar la profundidad del terreno con la ejecución de la apertura de calicatas y conservación de suelos. (Braja, M. 2001).

Las propiedades de los suelos así como el análisis de los mismos, se presentarán mediante el análisis de muestras de suelos en el laboratorio del sector de Vinchoa. Se obtendrá los porcentajes de arena, limo, arcilla, los mismos que nos servirán para identificar el tipo de textura del suelo mediante el triángulo de texturas, también se tendrá los valores de pH de suelos y la densidad. (Guaranda, G. 2017). 


\section{Zona de estudio}

La parroquia Veintimilla se ubica al este del cantón Guaranda, está bañada por ríos y quebradas; al oeste el río Guaranda y al este por las aguas de la cordillera Occidental; además recibe el aporte de quebradas que van a desembocar en el río Guaranda; como son las quebradas San Vicente, Llillohuaycu, y la Quinta.

Se ha tomado en cuenta para nuestro estudio las parroquias pertenecientes a la zona Gabriel Ignacio Veintimilla del cantón Guaranda, como es Vinchoa.

\section{Contexto geológico}

A continuación se describe las formaciones constituyentes del área de estudio:

Los volcánicos de Guaranda, conformados de materiales piroclásticos que están cubriendo la topografía preexistente, determina como tobas andesíticas de grano fino de color amarillo. Escorza (1993) denomina cobertura de la depresión de Guaranda.

La Unidad Macuchi.- Es una formación volcano sedimentario conformada por material volcánico de composición andesítica depositado en secuencias turbidíticas.

Los depósitos aluviales.-Son depósitos constituidos de arcillas, arenas.

\section{Materiales y métodos}

Para nuestro estudio se ha tomado en cuenta las parroquias pertenecientes a la zona Gabriel Ignacio Veintimilla del cantón Guaranda, como es Vinchoa. A continuación se indica los procesos aplicados en esta investigación.

Para realizar el análisis de suelos en el laboratorio se aplicaron los siguientes métodos:

\section{Método interno para texturas}

Este método nos permite identificar la textura del suelo y observar el contenido de partículas de arena, limo, arcilla.

\section{Textura:}

Para determinar la textura se aplicó el método interno, el que consta del siguiente procedimiento:

Equipos y Materiales:

Probeta de $250 \mathrm{ml}$

Agua

Agitador

Muestra de suelos

Procedimiento:

1) Tomar $100 \mathrm{~g}$ de suelo seco y colocarlo en la probeta de $250 \mathrm{ml}$. 
2) Adicionar $150 \mathrm{ml}$ de agua destilada hasta cubrir el suelo.

3) Con el agitador de vidrio revolver durante 10 minutos.

4) Dejar sedimentar por un tiempo de cinco días.

\section{Método del picnómetro}

Este método consiste en determinar la densidad real midiendo el peso de la muestra. Para esta se utiliza la siguiente fórmula:

pr $=($ Peso de la muestra $) /($ peso muestra - peso muestra en agua $)$

Peso muestra en agua $=$ (peso picnómetro muestra más agua) $-($ peso picnómetro muestra)

\section{Densidad:}

Para determinar la densidad se aplicó el método interno del picnómetro el que consta del siguiente procedimiento:

Equipos y Materiales:

Picnómetro $10 \mathrm{ml}$

Desecador de vacío

Bomba de vacío

Balanza analítica

Agua destilada

Embudo de plástico

Termómetro

Muestra de suelos

Procedimiento:

1) Pesar el picnómetro limpio y perfectamente seco.

2) Colocar en el interior del picnómetro $5 \mathrm{~g}$ de suelo usando un embudo plástico.

3) Anotar el peso del picnómetro con suelo, manteniendo el picnómetro completamente limpio (evitar humedad y/o grasa en las manos).

4) Adicionar agua destilada hasta la mitad del volumen del picnómetro; girar este entre los dedos con mucha suavidad y colocarlo en el desecador de vacío.

5) Dejar reposar dentro del desecador por 30 minutos aproximadamente y eliminar el vacío del desecador paulatinamente, secar el picnómetro y llenar hasta aforo con agua destilada, secar perfectamente el exterior y pesar en la balanza analítica.

6) Tomar la temperatura de la suspensión.

7) Vaciar el picnómetro, enjuagarlo perfectamente y llenarlo hasta aforo con agua destilada.

8) Pesar el picnómetro con agua y tomar la temperatura. 


\section{Método del potenciómetro (PEE/S/02 Ref: EPA9045 D)}

El método del potenciométrico o electroquímico para medir $\mathrm{pH}$ de un suelo es el más utilizado. Con este método se mide el potencial de un electrodo sensitivo a los iones $\mathrm{H}^{+}$(electrodo de vidrio) presentes en una solución problema; se usa como referencia un electrodo cuya solución problema no se modifica cuando cambia la concentración de los iones por medir, que es generalmente un electrodo de calomelano o de $\mathrm{Ag} / \mathrm{AgCl}$. El electrodo, a través de sus paredes, desarrolla un potencial eléctrico. En la práctica se utilizan soluciones amortiguadoras, de $\mathrm{pH}$ conocido, para calibrar el instrumento y luego comparar, ya sea el potencial eléctrico o el pH directamente de la solución por evaluar.

Las muestras que fueron recolectadas, normalmente deben recibir un tratamiento preliminar, antes de ser sometidas a algún tipo de técnica analítica, con el fin de estimar los contenidos de los elementos de interés.

Los objetivos de este tratamiento son:

Asegurarse que toda la muestra pueda transportarse y almacenarse adecuadamente.

Homogenizar la muestra de forma tal que la variabilidad en el submuestreo sea minimizado.

Realizar la separación preliminar de los constituyentes elementales de acuerdo a sus formas de ocurrencia en los diferentes materiales portadores.

Todos estos tratamientos se los realizó cuidadosamente con el fin de obtener una confiabilidad máxima en la identificación de todas las muestras y evitar la contaminación de estas.

En el laboratorio se realizaron análisis de suelo para determinar la textura, la densidad, $\mathrm{pH}$ del suelo.

Para los análisis nombrados anteriormente se utilizó el laboratorio de la Facultad de Ingeniería en Geología, Minas, Petróleos y Ambiental.

Las muestras para estos análisis se tomaron de las muestras superficiales en cinco lugares diferentes en el terreno que fueron extraídas.

\section{pH}

Para determinar el pH del suelo se aplicó el método del potenciómetro (PEE/S/02 Ref: EPA 9045 D) el que consta del siguiente procedimiento:

Equipos y Materiales:

Potenciómetro o medidor de $\mathrm{pH}$

Balanza A $\pm 0.01 \mathrm{~g}$

Vasos de precipitación de $50 \mathrm{ml}$ de vidrio

Vasos de precipitación de $250 \mathrm{ml}$ de vidrio

Varillas de vidrio

Embudos sin vástago

Papel filtro 
Papel absorbente

Muestra de suelos

Procedimiento:

1) Pesar $20 \mathrm{~g}$ de suelo y colocarlo en un vaso de precipitación de $50 \mathrm{ml}$.

2) Agregar $20 \mathrm{ml}$ de agua destilada, mezclar.

3) Agitar y dejar reposar 10 minutos.

4) Esperar una hora para la decantación.

5) Filtrar el contenido del vaso de precipitación con un embudo sin vástago y a través de un papel filtro. Si la separación de fases no es muy visible la mezcla y el $\mathrm{pH}$ se mide en la fase acuosa.

6) Pasados los 60 minutos, medir el $\mathrm{pH}$ con el potenciómetro.

\section{Para realizar el análisis de suelos en el campo se aplicaron los siguientes métodos:}

\section{Reconocimiento de la zona}

La morfología de la zona se caracteriza por presentar relieves no muy inclinados, predominando pendientes en el orden del $5 \%$ al $70 \%$.

Se ha evidenciado que geomorfológicamente la parroquia Veintimilla se caracteriza por la presencia de terrazas aluviales, relieves montañosos, comprende geográficamente la mayor parte de la cordillera Occidental, la que se presenta alargada en sentido norte-sur.

La parroquia Veintimilla está bańada por ríos y quebradas; al oeste el río Guaranda y al este por las aguas provenientes de la cordillera Occidental; además recibe el aporte de quebradas que van a desembocar en el río Guaranda; como son las quebradas San Vicente, Llillohuaycu, y la Quinta. (Guaranda, 2014).

\section{Apertura de calicatas}

La apertura de calicatas se realizó en el sector Vinchoa, observando que el material a extraer no sea producto de deslizamientos o acumulaciones de material al pie de monte.

Las dimensiones de la calicata son de $1.5 \mathrm{~m}$ de profundidad por $1 \mathrm{~m}$ a lo largo y ancho de la calicata.

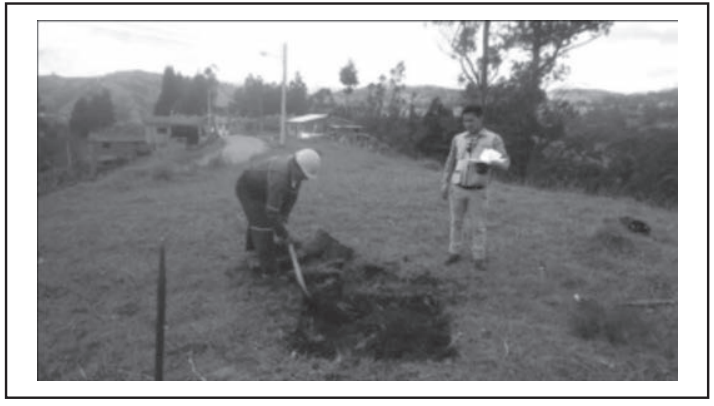

Figura 1. Apertura de calicatas. 
Los instrumentos que se utilizaron para la apertura de las calicatas fueron una barreta y una pala. El material extraído de la calicata era ubicado a un costado de la misma para luego de realizar el muestreo de suelos, proceder a tapar con el mismo material.

\section{Muestreo de suelos para análisis de laboratorio}

El muestreo de suelos se lo ejecuta una vez identificados los horizontes de suelos en la calicata. El muestreo se lo realiza desde la parte baja hasta la parte más alta de la calicata para que no exista contaminación al momento de realizar el muestreo.

El peso de cada muestra recolectada es de $1 \mathrm{~kg}$, el mismo que se coloca en una funda plástica, para luego ser procesado en el laboratorio para sus respectivos análisis.

Para el muestreo se utilizó:

- Espátula

- Fundas

- Flexómetro

Para complementar el muestreo de suelos de las calicatas, se realizaron dos excavaciones superficiales, con la finalidad de tomar muestras. Para este muestreo se quita la capa orgánica, y con el instrumento apropiado se muestrea un kilo de suelo.

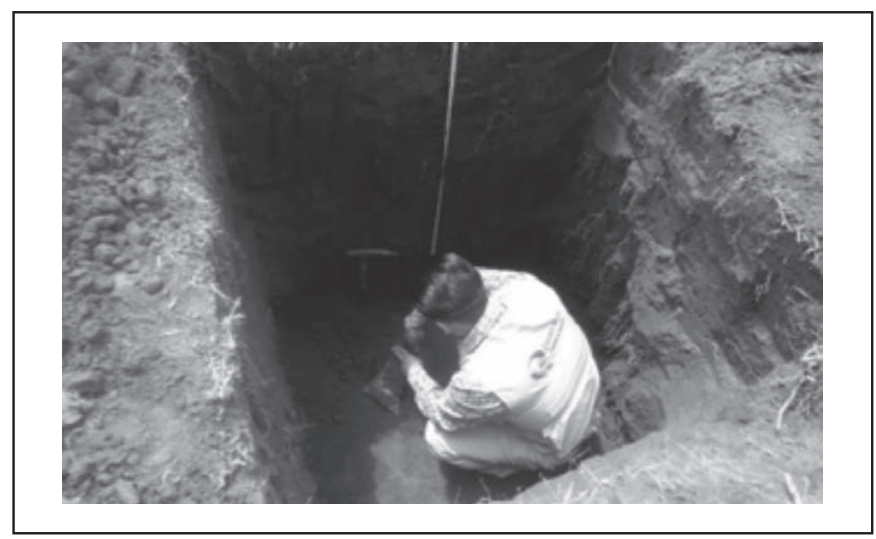

Figura 2. Muestreo de suelos.

\section{Resultados}

\section{Análisis de datos de campo y resultados de laboratorio}

Los datos de campo se colectaron de la apertura de calicatas y se identificó el número de horizontes del suelo. Se realizó la descripción de cada una de ellas las 
cuales fueron ejecutadas en la comunidad de Vinchoa, luego se correlaciona con los datos de laboratorio.

Para referencia del resultado de análisis de laboratorio se indica en la tabla 1 el análisis de resultados de la comunidad de Vinchoa.

Tabla 1. Análisis de resultados de la comunidad de Vinchoa

\begin{tabular}{|c|c|c|}
\hline Parámetros & Unidad & Valor \\
Densidad a $20^{\circ} \mathrm{C}$ & $\mathrm{g} / \mathrm{cm}^{3}$ & 2,24 \\
$\mathrm{pH}$ & $\mathrm{UpH}$ & 6,08 \\
\cline { 2 - 3 } Textura: Arena & $\%$ & 62,8 \\
\hline Textura: Limo & $\%$ & 36,0 \\
\hline Textura: Arcilla & $\%$ & 1,2 \\
\hline
\end{tabular}

Fuente: LABFIGEMPA

Para referencia del trabajo de campo ejecutado se indica en la tabla 2, la descripción de una de las calicatas de la comunidad de Vinchoa.

Tabla 2. Descripción de calicata de la comunidad de Vinchoa

\author{
Coordenadas UTM: \\ $\mathrm{X}=0724002$ \\ $Y=9822949$ \\ Cota: 2.696 m. s.n.m. \\ Profundidad de la calicata $(\mathrm{m}): 1.50$

\section{Descripción} \\ Horizonte A.- Materia orgánica, presencia de hojas y raíces \\ en estado de descomposición con un espesor de $15 \mathrm{~cm}$ apro- \\ ximadamente. \\ Horizonte B.- Presencia de clastos de $1-5 \mathrm{~cm}$. Existen clastos \\ menores a $30 \mathrm{~cm}$, de roca, se observa coloraciones negro, \\ café oscuro.
}

Fuente: Autor 
Tabla 3. Descripción de calicata de la Comunidad de Vinchoa

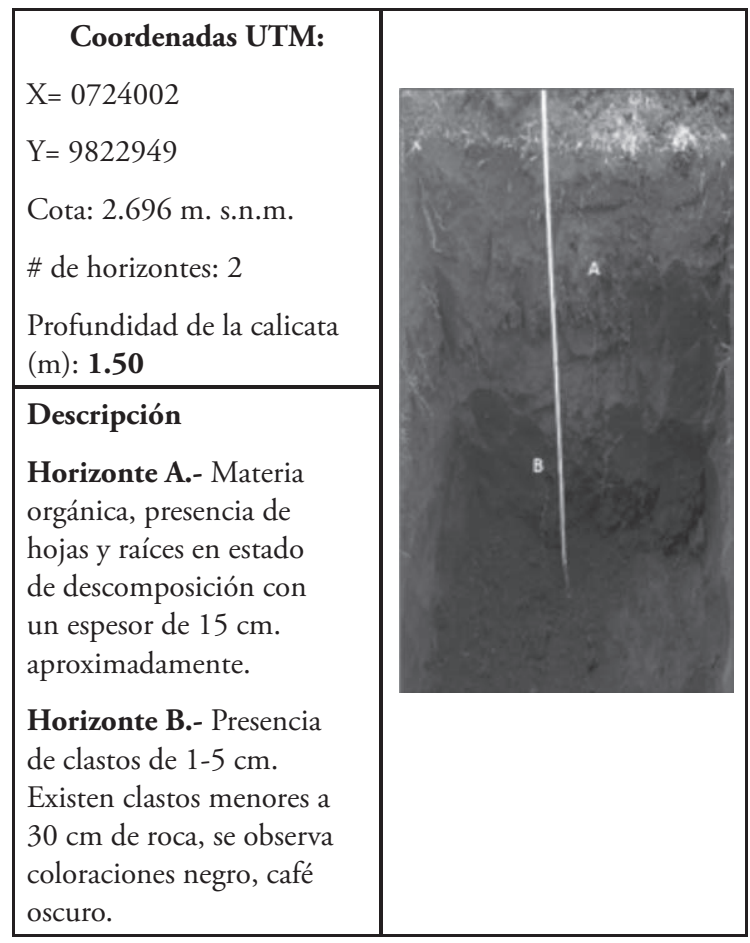

Fuente: Autor

El presente estudio se apoyó en los resultados de los datos de campo tomados de la descripción de los horizontes de la calicata y los resultados de laboratorio del análisis de muestras de suelos, los mismos que incluyen el porcentaje de arena, limo, arcilla, para identificar el tipo de textura del suelo.

Además se obtuvo el valor del $\mathrm{pH}$ y la densidad para identificar el tipo de $\mathrm{pH}$ del suelo en base al rango establecido por el Departamento de Agricultura de los Estados Unidos (USDA)

\section{Análisis de resultados texturas (Vinchoa)}

Del análisis de muestra realizado en el laboratorio del sector de Vinchoa se llegó a determinar porcentaje de arena de $62,8 \%$, de limo $36,0 \%$, de arcilla $1,2 \%$ lo cual nos da una textura de suelo franco arenoso por lo que podemos decir que la cantidad de los componentes del suelo se encuentra en proporciones óptimas o muy próximos a ella. Son suelos de elevada productividad, debido a su textura relativamente suelta, heredada de la arena, a su fertilidad procedente de limos incluidos y al mismo tiempo con adecuada retención de humedad por la arcilla presente. 
Análisis de resultados $\mathrm{pH}$, densidad (Vinchoa)

En base a la muestra del suelo se tiene un $\mathrm{pH}$ de 6,08, considerando un suelo ácido, debido a la escasez de bosques. Mediante el análisis de suelos se pudo obtener la densidad de $2,24 \mathrm{~g} / \mathrm{cm}^{3}$ a temperatura de $20^{\circ} \mathrm{C}$.

\section{Conclusiones}

El estudio de los análisis de suelos, demuestra que la mayoría de los peligros existentes en el área son de tipo antrópico, causantes de inestabilidad del terreno (movimientos en masa).

En el análisis efectuado se observó que además de la intervención antrópica, existen otros factores de riesgo para la zona y sus habitantes, estos pueden ser: las altas precipitaciones, las pendientes fuertes del terreno, las características de los suelos como los componentes inorgánicos y orgánicos, partículas de distinto tamaño: arena gruesa, limos y arcillas.

El uso agrícola del suelo y los asentamientos humanos en zonas de riesgos contribuyen al desencadenamiento de movimientos de remoción de masa, especialmente por el efecto de inestabilidad de los terrenos debido al uso inadecuado que se les da; tales como: cultivos no aptos, construcción de viviendas sin apego a la Norma Ecuatoriana de la Construcción.

En la zona de estudio se observa la presencia de monocultivos (pastos) y poca cantidad de montańa, esto hace que el suelo se vuelva ácido.

La zona de estudio presenta el tipo de suelo franco arenoso de baja plasticidad de consistencia rígida.

\section{Referencias}

Braja M. Das. (2001). Principios de ingeniería de cimentación. México: International Thomson Editores S.A.

Brizuela, L. A. (2013). Estudio de suelo.

Escorza, L. (1993). Levantamiento geológico de la depresión de Guaranda. Quito: Escorza, L.

Guaranda, G. (2014-11-14). Actualización del Plan de Desarrollo y Ordenamiento Territorial cantón Guaranda. Guaranda.

Guaranda, G. (3 de 3 de 2017). GADC Guaranda. Obtenido de GADC Guaranda: http://guaranda.gob.ec/newsiteCMT/cultura/ 